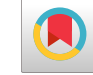

\title{
Acute Severe Bilateral Visual Loss as Presenting Symptom of COVID-19 Infection
}

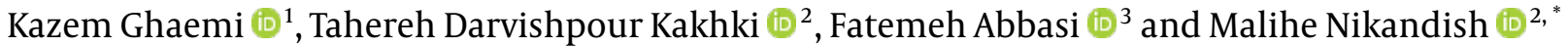 \\ ${ }^{1}$ Department of Neurosurgery, School of Medicine, Cellular and Molecular Research Center, Birjand University of Medical Sciences, Birjand, Iran \\ ${ }^{2}$ Ophthalmologist, Department of Ophthalmology, Razi Hospital, Birjand University of Medical Sciences, Birjand, Iran \\ ${ }^{3}$ Research Committee, Birjand University of Medical Sciences, Birjand, Iran \\ "Corresponding author: Razi Hospital, Birjand, Iran. Email: nikandishm@bums.ac.ir
}

Received 2021 August 07; Revised 2021 September 03; Accepted 2021 September 07.

\begin{abstract}
Introduction: Coronavirus disease 2019 (COVID-19) has emerged as a pandemic and is accompanied by extraordinary morbidity and mortality. Critically ill COVID-19 patients have frequent thrombotic problems and laboratory evidence of hypercoagulability. The spectrum of presentations and complications of COVID-19 is still evolving. We describe a patient with unusual visual symptoms as the presenting signs of COVID-19.

Case Presentation: In this case report, we describe a patient who presented with acute severe bilateral visual loss and headache. On neurological examination, cranial nerves were normal. There was no motor and sensorial abnormality. While the patient was in the emergency room, he was tested positive for COVID-19 via nasopharyngeal swab polymerase chain reaction (PCR) as part of COVID-19 surveillance. An unenhanced brain CT scan showed infarction in the occipital cortex bilaterally due to ischemic stroke involving the Posterior Cerebral Artery (PCA). A few hours later, oxygen saturation was 42\%, and a sudden decline of consciousness was observed, progressing to coma. The neurosurgery intervention failed to change the patient's status, and he was pronounced dead in the next few hours.

Conclusions: This case illuminates a wide range of COVID-19-related symptomatology and highlights the need for clinicians to be aware of different clinical appearances associated with this infection.
\end{abstract}

Keywords: COVID-19, Vision Loss, Stroke, Ophthalmology

\section{Introduction}

Coronavirus disease 2019 (COVID-19) was first reported in Wuhan, China, in December 2019 (1). While the most common symptoms of COVID-19 are fever, cough, and shortness of breath, neurologic complications have ascended as a progressively recognized area of morbidity and mortality (2). In the adult population, the nervous system involvement is more likely in severe cases than in non-severe patients. However, neurologic manifestations have also been reported in patients without the typical features of COVID-19 (3). Neurologic manifestations in COVID19 may be nonspecific or specific. More specific manifestations related to COVID-19 are also observed, such as the impairment of smell or taste, acute cerebrovascular disease, and seizure (4). The ocular manifestations of COVID19 have a broad spectrum, from conjunctivitis and anterior uveitis to sight-threatening conditions like retinitis and optic neuritis (5). We report a case of COVID-19 patient resulting in ICU admission and eventually death, presenting with severe bilateral visual loss and ischemia in the posterior cerebral artery (PCA) territory as the initial symptoms before typical respiratory involvement.

\section{Case Presentation}

A man in his 70s presented with acute, painless, severe binocular visual loss from a few hours ago. He had no complaints other than minimal headache. The study protocol and examinations were reviewed and approved by the Ethics Committee of Birjand University of Medical Sciences. Written informed consent was obtained from the patient's relatives for publishing the patient information and details of his disease.

In the emergency room, the patient's vital signs were as follows: Temperature $37^{\circ} \mathrm{C}$, blood pressure $140 / 88 \mathrm{mmHg}$, respiratory rate 17 breaths/min, and oxygen saturation $98 \%$. Ophthalmological examination showed visual acuity of hand motion in both eyes. Anterior and posterior segments and motility examination were normal; no periph- 
eral retinal hemorrhages or emboli were noted, and the relative afferent pupillary defect was absent. Given his normal ophthalmologic exam and bilateral profound visual loss, the preliminary diagnosis of cerebral blindness was considered to be more likely. Hence, the patient was hospitalized, and we consulted a neurologist.

In neurological examination, cranial nerves were normal. There was no motor and sensorial abnormality. His laboratory parameters, including complete blood count, blood sugar and lipid profile, as well as renal and liver function test, electrolytes, and cardiac enzymes, were normal. An electrocardiogram showed a normal sinus rhythm. Prothrombin time [15.3 $(14.4-16.3)]$, activated partial thromboplastin time [28.4 (40.2 - 51.0)], and D-dimer levels were normal. An unenhanced brain CT scan showed infarction in the occipital cortex bilaterally due to ischemic stroke involving the PCA (Figure 1). While the patient was in the emergency room, he was tested positive for COVID-19 via nasopharyngeal swab polymerase chain reaction (PCR) as part of COVID-19 surveillance. A few hours after hospitalization, the patient was distinguished to be febrile with a temperature of $39^{\circ} \mathrm{C}$ and CRP of 9.9. The chest $\mathrm{X}$-ray showed a ground-glass appearance on the left side and the upper two-thirds of the right side (Figure 2). Within a few hours, blood oxygen saturation dropped rapidly, and the patient showed loss of consciousness. Severe ischemia in PCA territory resulted in hydrocephaly. A few hours later, oxygen saturation was $42 \%$, and a sudden decline of consciousness was observed, progressing to coma. The neurosurgery intervention failed to change the patient's status, and he was pronounced dead in the next few hours.

\section{Discussion}

The spectrum of presentations and complications of COVID-19 is still evolving. Our patient presented with visual symptoms and without any systemic signs or symptoms. Then, within a few hours, an increase in body temperature and a decrease in arterial saturation and lung involvement were established, and eventually, the patient died due to a severe decrease in blood oxygen level as a COVID-19 complication.

The frequency of thrombotic events is high in patients with COVID-19 contamination that is associated with poorer outcomes. Common causes of acute bilateral vision loss include occipital infarction, traumas of the occipital lobe, migraine, occipital seizures, aggressive cardiac processes, and preeclampsia. Bilateral occipital infarct prompted by Stroke is a rare condition, and its diagnosis may be challenging due to unusual symptoms (6). Prompt diagnosis is very imperative in all kinds of ischemia. Among admitted COVID-19 patients, neurologic

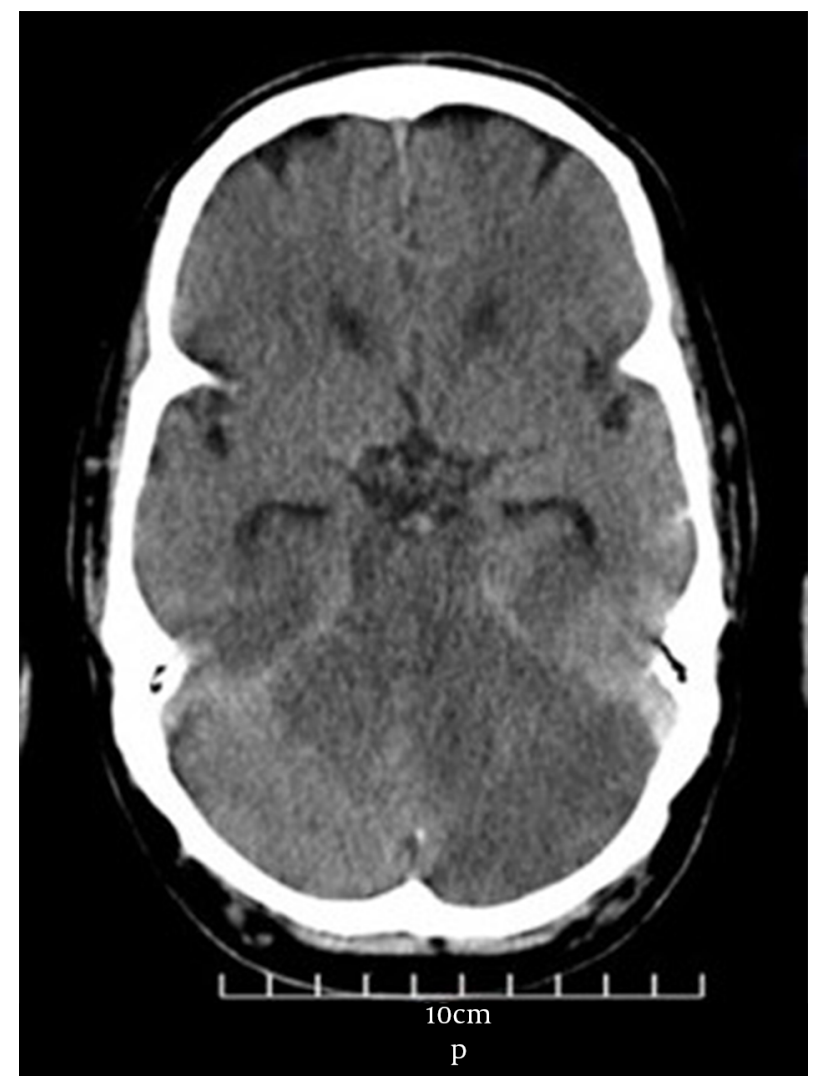

Figure 1. Unenhanced brain CT scan showing infarction in the occipital cortex bilaterally due to ischemic stroke involving the posterior cerebral artery.

problems range from 6 to 36\% (7). The explained mechanisms of CNS involvement in COVID-19 include direct infection through angiotensin-converting enzyme 2 (ACE-2) receptors, neuroinflammation, post-viral triggered autoimmune response, hypercoagulability, and metabolic or hypoxic injury (8). Recent literature has shown the association between COVID-19 infection and derangement in the coagulation profile (9). Some studies showed associations between critically ill COVID-19 patients and thrombotic complications (10). The coagulopathy that COVID-19 patients show may be distinctive and different from other virus-induced or sepsis-related coagulopathy, and extensive research remains to be done. A possible explanation for the occurrence of coagulation disorders in COVID-19 patients is the enhanced thrombus formation under hypoxia conditions (11). Local inflammation and a vasculitic process in arterial walls after COVID-19 infection can result in vasoconstriction with subsequent organ ischemia, inflammation associated with tissue edema, and procoagulant state (12). Several studies showed ischemic stroke secondary to the occlusion of large vessels in COVID-19 infec- 


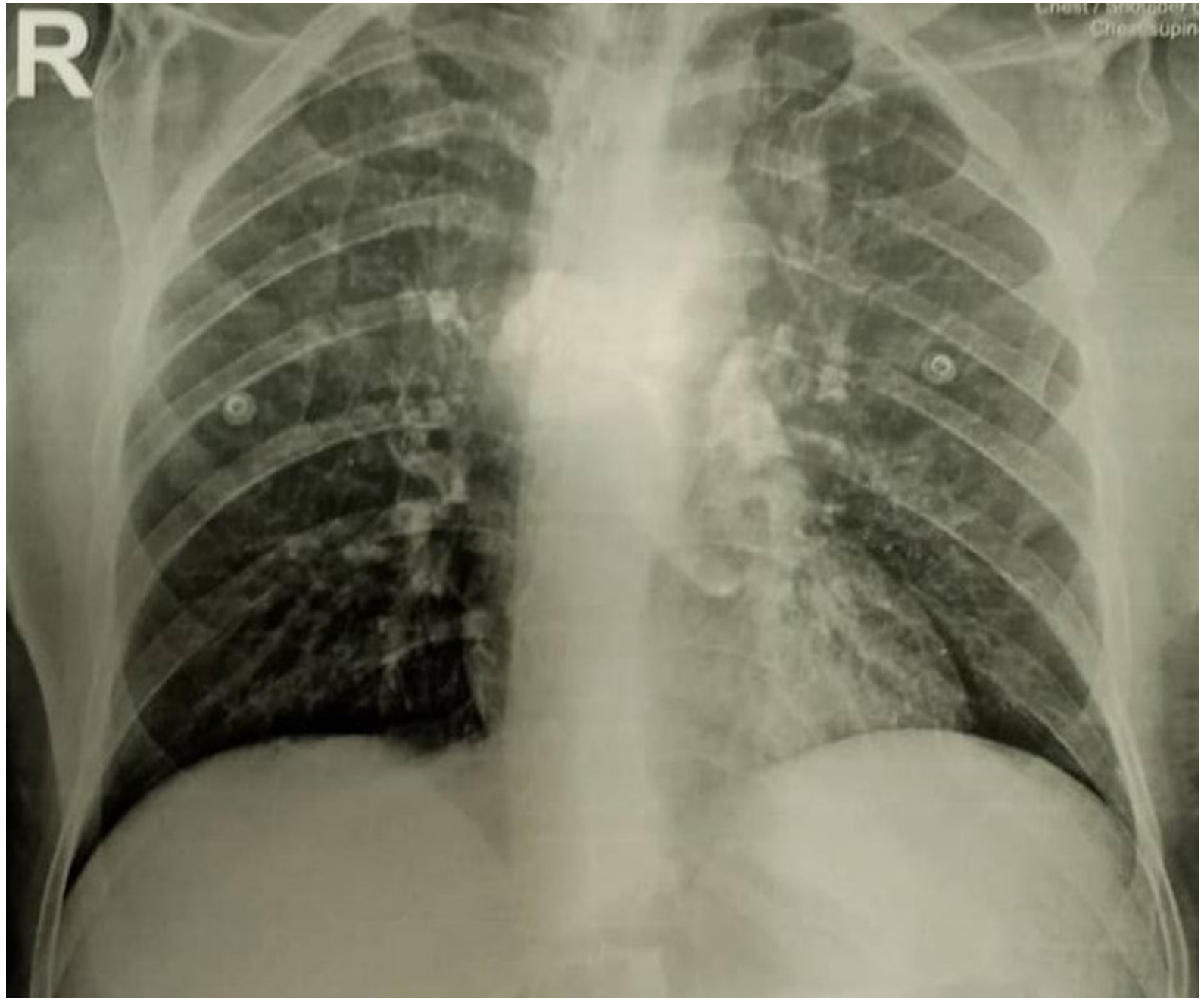

Figure 2. Chest X-ray showing a ground-glass appearance on the left side and the upper two-thirds of the right side.

tion. Although PCA involvement is not common, it can occur less frequently (13). Giving the normal value of coagulation profile and apparent lack of other vascular risk factors in the present case, we considered vasculopathy secondary to COVID-19 infection as a cause of ischemia in the PCA. To our knowledge, this is the first case of COVID-19 that presented with acute bilateral visual loss secondary to PCA vasculopathy.

\subsection{Conclusion}

This case clarifies the need to suspect COVID-19 infection in patients presenting visual symptoms secondary to ischemia such as acute vision loss, which includes patients who are asymptomatic or minimally symptomatic for COVID-19 infection. This case also illuminates a wide range of COVID-19-related symptomatology and highlights the need for clinicians to be aware of different clinical appearances associated with this infection.

\section{Footnotes}

Authors' Contribution: All authors contributed to the study, prepared the manuscript for publication equally, read and confirmed the final manuscript for publication.

Conflict of Interests: No conflict of interest exists in relation to the submitted manuscript.

Ethical Approval: The study protocol and examinations were reviewed and approved by the Ethics Committee of Birjand University of Medical Sciences (IR.BUMS.REC.1399.495). 
Funding/Support: The authors received no specific funding for this report.

Informed Consent: Written informed consent was obtained from the patient's relatives for publishing the patient's information and details of his disease.

\section{References}

1. Tham AC, Thein TL, Lee CS, Tan GSE, Manauis CM, Siow JK, et al. Olfactory taste disorder as a presenting symptom of COVID19: A large single-center Singapore study. Eur Arch Otorhinolaryngol. 2021;278(6):1853-62. doi: 10.1007/s00405-020-06455-0. [PubMed: 33159556]. [PubMed Central: PMC7648204].

2. Singh J, Ali A. Headache as the presenting symptom in 2 patients with COVID-19 and a history of migraine: 2 case reports. Headache. 2020;60(8):1773-6. doi: 10.1111/head.13890. [PubMed: 32521062]. [PubMed Central: PMC7300704].

3. Kim Y, Walser SA, Asghar SJ, Jain R, Mainali G, Kumar A. A comprehensive review of neurologic manifestations of COVID-19 and management of pre-existing neurologic disorders in children. J Child Neurol. 2021;36(4):324-30. doi: 10.1177/0883073820968995. [PubMed: 33112694]. [PubMed Central: PMC7859660].

4. Pinzon RT, Wijaya VO, Buana RB, Al Jody A, Nunsio PN. Neurologic characteristics in Coronavirus disease 2019 (COVID-19): A systematic review and meta-analysis. Front Neurol. 2020;11:565. doi: 10.3389/fneur.2020.00565. [PubMed: 32574250]. [PubMed Central: PMC7273516].

5. Ortiz-Seller A, Martinez Costa L, Hernandez-Pons A, Valls Pascual E, Solves Alemany A, Albert-Fort M. Ophthalmic and neuro-ophthalmic manifestations of Coronavirus disease 2019 (COVID-19). Ocul Immunol Inflamm. 2020;28(8):1285-9. doi: 10.1080/09273948.2020.1817497. [PubMed: 33021422].
6. Tanrikulu CS, Hocagil H, Kaya U, Hocagil AC. Acute bilateral vision loss in emergency department: A case report. Turk J Emerg Med. 2016;16(1):38-40. doi: 10.1016/j.tjem.2014.12.001. [PubMed: 27239639]. [PubMed Central: PMC4882198].

7. Bridwell R, Long B, Gottlieb M. Neurologic complications of COVID-19. Am J Emerg Med. 2020;38(7):1549 e3-7. doi: 10.1016/j.ajem.2020.05.024. [PubMed: 32425321]. [PubMed Central: PMC7229718].

8. Keyhanian K, Umeton RP, Mohit B, Davoudi V, Hajighasemi F, Ghasemi M. SARS-CoV-2 and nervous system: From pathogenesis to clinical manifestation. J Neuroimmunol. 2020;350:577436. doi: 10.1016/j.jneuroim.2020.577436. [PubMed: 33212316]. [PubMed Central: PMC7647896]

9. Hendaus MA, Jomha FA. From COVID-19 to clot: The involvement of the complement system. J Biomol Struct Dyn. 2020:1-6. doi: 10.1080/07391102.2020.1832919. [PubMed: 33063628].

10. Roh DJ, Eiseman K, Kirsch H, Yoh N, Boehme A, Agarwal S, et al Hypercoagulable viscoelastic blood clot characteristics in critically ill Coronavirus disease 2019 patients and associations with thrombotic complications. J Trauma Acute Care Surg. 2021;90(1):e7-e12. doi: 10.1097/TA.0000000000002963. [PubMed: 33009340].

11. Harapan BN, Yoo HJ. Neurological symptoms, manifestations, and complications associated with severe acute respiratory syndrome Coronavirus 2 (SARS-CoV-2) and Coronavirus disease 19 (COVID19). J Neurol. 2021;268(9):3059-71. doi: 10.1007/s00415-021-10406-y. [PubMed: 33486564]. [PubMed Central: PMC7826147].

12. Varga Z, Flammer AJ, Steiger P, Haberecker M, Andermatt R, Zinkernagel AS, et al. Endothelial cell infection and endotheliitis in COVID19. Lancet. 2020;395(10234):1417-8. doi:10.1016/S0140-6736(20)30937-5. [PubMed: 32325026]. [PubMed Central: PMC7172722].

13. Robles LA. Bilateral large vessel occlusion causing massive ischemic stroke in a COVID-19 patient. J Stroke Cerebrovasc Dis. 2021;30(3):105609. doi: 10.1016/j.jstrokecerebrovasdis.2021.105609. [PubMed: 33429238]. [PubMed Central: PMC7791307]. 